\title{
HST ROTATIONAL SPECTRAL MAPPING OF TWO L-TYPE BROWN DWARFS: VARIABILITY IN AND OUT OF WATER BANDS INDICATES HIGH-ALTITUDE HAZE LAYERS
}

\author{
Hao Yang ${ }^{1}$, Dániel Apai ${ }^{1,2}$, Mark S. Marley ${ }^{3}$, Didier Saumon ${ }^{4}$, Caroline V. Morley $^{5}$, Esther Buenzli ${ }^{6}$, \\ Étienne Artigau ${ }^{7}$, Jacqueline Radigan $^{8}$, Stanimir Metchev ${ }^{9}$, Adam J. Burgasser ${ }^{10}$, Subhanjoy Mohanty ${ }^{11}$, \\ Patrick J. Lowrance ${ }^{12}$, Adam P. Showman ${ }^{2}$, Theodora Karalidi ${ }^{1}$, Davin Flateau ${ }^{2}$, and Aren N. Heinze ${ }^{13}$ \\ ${ }^{1}$ Department of Astronomy, University of Arizona, 933 North Cherry Avenue, Tucson, AZ 85721, USA; haoyang @ email.arizona.edu \\ ${ }^{2}$ Department of Planetary Sciences, University of Arizona, 1629 East University Boulevard, Tucson, AZ 85721, USA \\ ${ }^{3}$ NASA Ames Research Center, Naval Air Station, Moffett Field, Mountain View, CA 94035, USA \\ ${ }^{4}$ Los Alamos National Lab, Los Alamos, NM 87545, USA \\ ${ }^{5}$ Department of Astronomy and Astrophysics, University of California, Santa Cruz, CA 95064, USA \\ ${ }^{6}$ Max-Planck-Institut für Astronomie, Königstuhl 17, D-69117 Heidelberg, Germany \\ ${ }^{7}$ Département de Physique, Université de Montréal, C.P. 6128 Succ. Centre-ville, Montréal, QC H3C 3J7, Canada \\ ${ }^{8}$ Space Telescope Science Institute, 3700 San Martin Drive, Baltimore, MD 21218, USA \\ ${ }^{9}$ Department of Physics and Astronomy, Western University, 1151 Richmond Street, London, ON N6A 3K7, Canada \\ ${ }^{10}$ Center for Astrophysics and Space Science, University of California San Diego, La Jolla, CA 92093, USA \\ ${ }^{11}$ Imperial College London, 1010 Blackett Lab, Prince Consort Road, London SW7 2AZ, UK \\ 12 Infrared Processing and Analysis Center, MS 100-22, California Institute of Technology, Pasadena, CA 91125, USA \\ ${ }^{13}$ Department of Physics and Astronomy, State University of New York, Stony Brook, NY 11794-3800, USA \\ Received 2014 October 23; accepted 2014 November 7; published 2014 December 17
}

\begin{abstract}
We present time-resolved near-infrared spectroscopy of two L5 dwarfs, 2MASS J18212815+1414010 and 2MASS J15074759-1627386, observed with the Wide Field Camera 3 instrument on the Hubble Space Telescope (HST). We study the wavelength dependence of rotation-modulated flux variations between $1.1 \mu \mathrm{m}$ and $1.7 \mu \mathrm{m}$. We find that the water absorption bands of the two L5 dwarfs at $1.15 \mu \mathrm{m}$ and $1.4 \mu \mathrm{m}$ vary at similar amplitudes as the adjacent continuum. This differs from the results of previous $H S T$ observations of L/T transition dwarfs, in which the water absorption at $1.4 \mu \mathrm{m}$ displays variations of about half of the amplitude at other wavelengths. We find that the relative amplitude of flux variability out of the water band with respect to that in the water band shows a increasing trend from the L5 dwarfs toward the early T dwarfs. We utilize the models of Saumon \& Marley and find that the observed variability of the L5 dwarfs can be explained by the presence of spatially varying high-altitude haze layers above the condensate clouds. Therefore, our observations show that the heterogeneity of haze layers-the driver of the variability - must be located at very low pressures, where even the water opacity is negligible. In the near future, the rotational spectral mapping technique could be utilized for other atomic and molecular species to probe different pressure levels in the atmospheres of brown dwarfs and exoplanets and uncover both horizontal and vertical cloud structures.
\end{abstract}

Key words: brown dwarfs - stars: atmospheres - stars: individual (2MASS J18212815+1414010, 2MASS

J15074769-1627386, 2MASS J01365662+0933473) - stars: low-mass

\section{INTRODUCTION}

Since the recent discovery of three early $\mathrm{T}$ dwarfs with highamplitude flux variability as large as $26 \%$ in the near-infrared (near-IR; Artigau et al. 2009; Radigan et al. 2012; Biller et al. 2013), ground- and space-based observations have revealed that low-level variabilities are common for brown dwarfs of diverse spectral types across a wide range of wavelengths (e.g., Heinze et al. 2013; Gillon et al. 2013; Buenzli et al. 2014; Burgasser et al. 2014; Wilson et al. 2014; Radigan et al. 2014; Radigan 2015; Metchev et al. 2015).

Condensate clouds are believed to play a major role in the atmospheres of brown dwarfs (e.g., Tsuji et al. 1996; Jones \& Tsuji 1997; Burrows et al. 2000; Allard et al. 2001; Ackerman \& Marley 2001; Tsuji 2002; Helling et al. 2008; Saumon \& Marley 2008; Stephens et al. 2009), and heterogeneous cloud covers combined with fast rotation are thought to produce the observed flux variability. For the $\mathrm{L}$ dwarfs, silicate cloud layers form from condensation and strongly impact the emergent flux at these effective temperatures (e.g., Chabrier et al. 2000; Lodders $\&$ Fegley 2006). Mid and late T dwarfs are regarded as generally cloud-free objects due to clouds dispersing or sinking below their photospheres (e.g., Ackerman \& Marley 2001; Burgasser et al. 2002), though sulfide clouds might still exist at altitudes high enough to affect the atmosphere (Morley et al. 2012). As transitional objects between $\mathrm{L}$ dwarfs and mid/late $\mathrm{T}$ dwarfs, early $\mathrm{T}$ dwarfs show intermediate near-IR colors. Based on an emerging number of variable brown dwarfs, these objects likely have heterogeneous cloud coverage (e.g., Apai et al. 2013; Radigan et al. 2014; Crossfield et al. 2014; Buenzli et al. 2014, 2015), caused by varying cloud thickness, i.e., thin-thick clouds, but no cloud holes (Radigan et al. 2012; Apai et al. 2013). Recent models have also shown that temperature perturbations, potentially arising due to atmospheric circulation, could also induce periodic and aperiodic flux variations (Showman \& Kaspi 2013; Zhang \& Showman 2014; Robinson \& Marley 2014; Morley et al. 2014).

Studies of clouds are not only important in understanding brown dwarf atmospheres, but also can shed light on the atmospheric properties of exoplanets (Kostov \& Apai 2013). Recent observations of transiting exoplanets show that high-altitude clouds or haze layers may exist at pressure levels of $1 \mathrm{mbar}$ or even lower (e.g., Sing et al. 2011; Kreidberg et al. 2014; Knutson et al. 2014). While more detailed characterization of 
Table 1

Journal of Observations

\begin{tabular}{|c|c|c|c|c|c|c|c|c|}
\hline Target & Full Name & $\begin{array}{l}\text { Spectral } \\
\text { Type }\end{array}$ & $\begin{array}{c}J \\
\text { Mag. }\end{array}$ & Date & $\begin{array}{l}\text { Exposure } \\
\text { Time (s) }\end{array}$ & $\begin{array}{c}N_{\exp } \\
\text { per Orbit }\end{array}$ & $\begin{array}{c}\text { No. of Orbits } \\
\times \text { Visits }\end{array}$ & Reference \\
\hline 2M1507 & 2MASS J15074769-1627386 & L5 & 12.83 & 2013 Apr 30 \& May 12 & 67.30 & 30 & $4 \times 2$ & Reid et al. (2000) \\
\hline 2M1821 & 2MASS J18212815+1414010 & L5 & 13.43 & 2013 Jun 9 \& Jun 27 & 112.00 & 19 & $3 \times 2$ & Looper et al. (2008) \\
\hline SIMP0136 & 2MASS J01365662+0933473 & $\mathrm{T} 2.5$ & 13.45 & 2013 Sep $28 \&$ Oct 7 & 112.00 & 19 & $4 \times 2$ & Artigau et al. (2006) \\
\hline
\end{tabular}

the exoplanetary atmospheres is limited by current instrumentation, studies of clouds or haze layers in brown dwarf atmospheres offer essential insights for reference.

Rotational spectral mapping is a powerful technique for uncovering cloud structures on brown dwarfs. Buenzli et al. (2012) utilized the unique capabilities of the Wide Field Camera 3 (WFC3) instrument on the Hubble Space Telescope (HST) and detected phase shifts among light curves of different wavelength bands in the T6 dwarf 2M22282889-4310262. The multi-layer rotational maps revealed heterogeneous atmospheric structures in both horizontal and vertical directions for the first time. Apai et al. (2013) analyzed high-precision time-resolved WFC3 spectra of two L/T transition dwarfs, 2MASS J21392676+0220226 (hereafter 2M2139) and 2MASS J01365662+0933473 (hereafter SIMP0136; Artigau et al. 2006). Modeling the spectral and color changes showed that explaining the brightness variations requires a combination of thick and thin clouds. Burgasser et al. (2014) monitored WISE J104915.57-531906.1 (or Luhman 16, Luhman 2013) both photometrically and spectroscopically, and were able to reproduce the observed spectral variability with a brightness temperature two-spot model. By analyzing HST/WFC3 spectral time series, Buenzli et al. (2015) found that Luhman 16B varies at all wavelengths from 1.1 to $1.6 \mu \mathrm{m}$ with amplitudes ranging from $7 \%$ to $11 \%$, and a two-component thin-thick cloud model could explain most of the variability. So far, this technique has been applied to mostly T dwarfs and only very short ( $\sim 40$ minutes) time series of a few L dwarfs without covering a full rotation in an HST snapshot survey (Buenzli et al. 2014) are available. Thus, there is currently limited observational information of time-variable components of the mean atmospheric structure of L dwarfs.

In this Letter, we report HST/WFC3 spectral mapping of two L5 dwarfs, 2MASS J18212815+1414010 and 2MASS J15074769-1627386 (hereafter 2M1821 and 2M1507). 2M1821 was discovered by Looper et al. (2008) and exhibits red near-IR colors $(J-K=1.78)$ and silicate absorption at 9-11 $\mu \mathrm{m}$ (Cushing et al. 2006), which they concluded was indicative of unusually large dust opacity in the atmosphere, possibly due to low surface gravity or high metallicity. Gagné et al. (2014) identified 2M1821 as a young field dwarf that shows signs of low surface gravity. 2M1507 was discovered by (Reid et al. 2000) and also shows weak 9-11 $\mu \mathrm{m}$ silicate absorption (Cushing et al. 2006). Both L5 dwarfs have been observed to be variable in Spitzer IRAC channels 1 and 2 (Metchev et al. 2015). We study the wavelength dependence of their near-IR variabilities, and compare the results with early T dwarfs, 2M2139, SIMP0136, and Luhman 16B. In Section 2, we describe our observations and the data reduction process. In Section 3, we present the spectral variation of the two L dwarfs, which is followed by a discussion of the results in Section 4. We summarize our results in Section 5.

\section{OBSERVATIONS AND DATA REDUCTION}

The Spitzer Space Telescope Cycle-9 Exploration Science Program, Extrasolar Storms (PI: D. Apai), uses coordinated multi-epoch HST and Spitzer rotational phase maps of six brown dwarfs to characterize cloud evolution and dynamics of ultracool atmospheres over a large range of timescales. The observations presented here are part of the coordinated HST component of the Extrasolar Storms program. We obtained near-IR spectra of 2M1821, 2M1507, and the T2 dwarf SIMP0136 with the WFC3 G141 grism.

Each target in our program was observed over three or four consecutive orbits each in two separate visits. During each orbit, a direct image was first obtained through the F132N filter for wavelength calibration, followed by a number of dispersed images with the G141 grism. To avoid detector buffer dumps and maximize observing time in each orbit, subarrays of $256 \times$ 256 pixels on the detector were used, corresponding to a field of view of $\sim 30^{\prime \prime} \times 30^{\prime \prime}$. The spectra were kept on the same pixels for all exposures so that systematic errors caused by pixel-topixel sensitivity variations are avoided. The observations are summarized in Table 1.

For data reduction, we downloaded spectral images processed by the standard WFC3 pipeline from the MAST archive, ${ }^{14}$ and then utilized custom IDL routines and the PyRAF software package aXe $\mathrm{X}^{15}$ to extract the slitless spectra. The detailed data reduction process is described in Apai et al. (2013) and Buenzli et al. (2014). Briefly, in the two-dimensional spectral images (.flt files) already processed by the WFC3 pipeline, we first corrected cosmic rays and bad pixels flagged by the pipeline. Then we embedded the subarray images into full-frame ones so that aXe can use full-frame standard instrument calibration images. The axeprep routine was used to subtract sky background before the axecore routine was applied to extract the spectra with a fixed 8-pixel extraction window. The reduced G141 grism spectra provide a wavelength coverage of $1.05-1.7 \mu \mathrm{m}$ and a spectral resolution of $\sim 130$. The uncertainty level (including photon noise, readout and background noise) for our observations is about $0.3 \%$ and is estimated using the observed spectra and the WFC3 IR Spectroscopic Exposure Time Calculator (ver.: 22.1.2).

Archival HST/WFC3 observations of SIMP0136 and the T2.5 dwarf 2M2139 from GO program 12314 (PI: D. Apai) were also downloaded for comparison purposes and reduced in the same fashion described above.

During the first orbit in each visit, there was a common steep increase in brightness due to a systematic ramp effect. This is exemplified by the $J$-band light curve of a non-variable reference star in the field of view of 2M1821 (Figure 1). The $J$-band fluxes are calculated by integrating each spectrum convolved with the 2MASS $J$-band spectral response curve (Cohen et al. 2003). The first orbit shows a flux increase of nearly $1 \%$, while the second and third orbits shown stable flux levels within uncertainty $(\sim 0.3 \%)$. As discussed in Apai et al. (2013) and Buenzli et al. (2014), the ramp is found to be largely independent of wavelength and of object brightness. To remove the ramp

\footnotetext{
14 http://archive.stsci.edu

15 http://axe-info.stsci.edu
} 


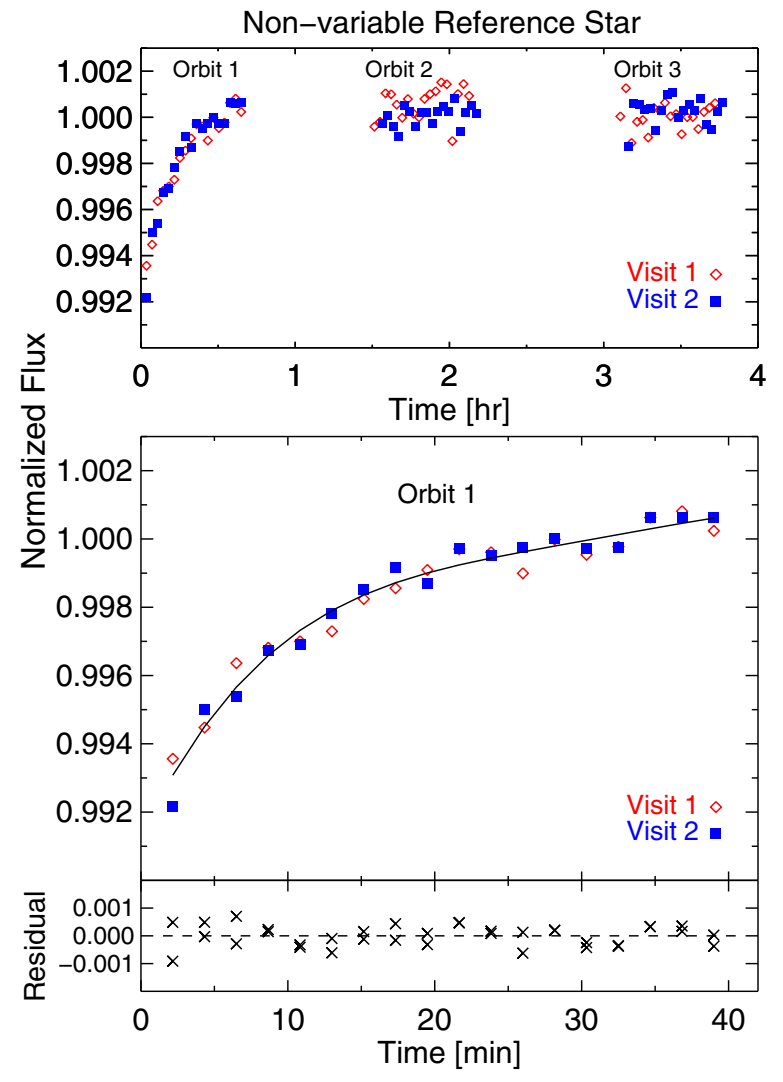

Figure 1. Correction of the ramp based on $J$-band light curves of a non-variable reference star. Top: $J$-band light curves of the reference stars from two HST visits. Each visit has three orbits of observations. The first orbits show an increasing ramp, while the second and third orbits have no substantial flux variation above the uncertainty level. Bottom: the ramp in the first orbits is fitted with a fourth-order polynomial function, and the residuals after applying the polynomial correction have a standard deviation of 0.00038 .

effect, we fit a fourth-order polynomial function to the light curves of the first orbits for the non-variable reference star (bottom panels of Figure 1), and applied the correction to the first-orbit observations of the variable brown dwarfs.

\section{RESULTS}

Our spectral time series of 2M1821 and 2M1507 reveal brightness variations between $1.1 \mu \mathrm{m}$ and $1.7 \mu \mathrm{m}$. In Figure 2, we show their brightest and faintest spectra from respective $H S T$ Visit 1. Also shown for comparison are the spectra of the T2 dwarf SIMP0136 and re-reduced archival data of the T2.5 dwarf 2M2139. The spectra of both the L5 dwarfs and early T dwarfs exhibit prominent absorption features of alkali elements and water. The L5 dwarfs have stronger $\mathrm{Na}$ I and $\mathrm{K}$ I absorption lines, while the early $\mathrm{T}$ dwarfs show deeper water absorption bands near 1.15 and $1.4 \mu \mathrm{m}$ regions along with methane absorption features.

We compare the ratios of the brightest and faintest spectra in an HST visit and discover that the variation of water-band absorption around $1.4 \mu \mathrm{m}$ behaves differently for the two L5 dwarfs and the two L/T transition dwarfs. Apai et al. (2013) first discovered for SIMP0136 and 2M2139 that the water band around $1.4 \mu \mathrm{m}$ varies at a reduced amplitude compared to the continuum and other atomic and molecular absorption features. The same reduced water-band variability is also found for Luhman 16B (Buenzli et al. 2015). However, for the two L5 dwarfs, we find that the ratio of the brightest over faintest

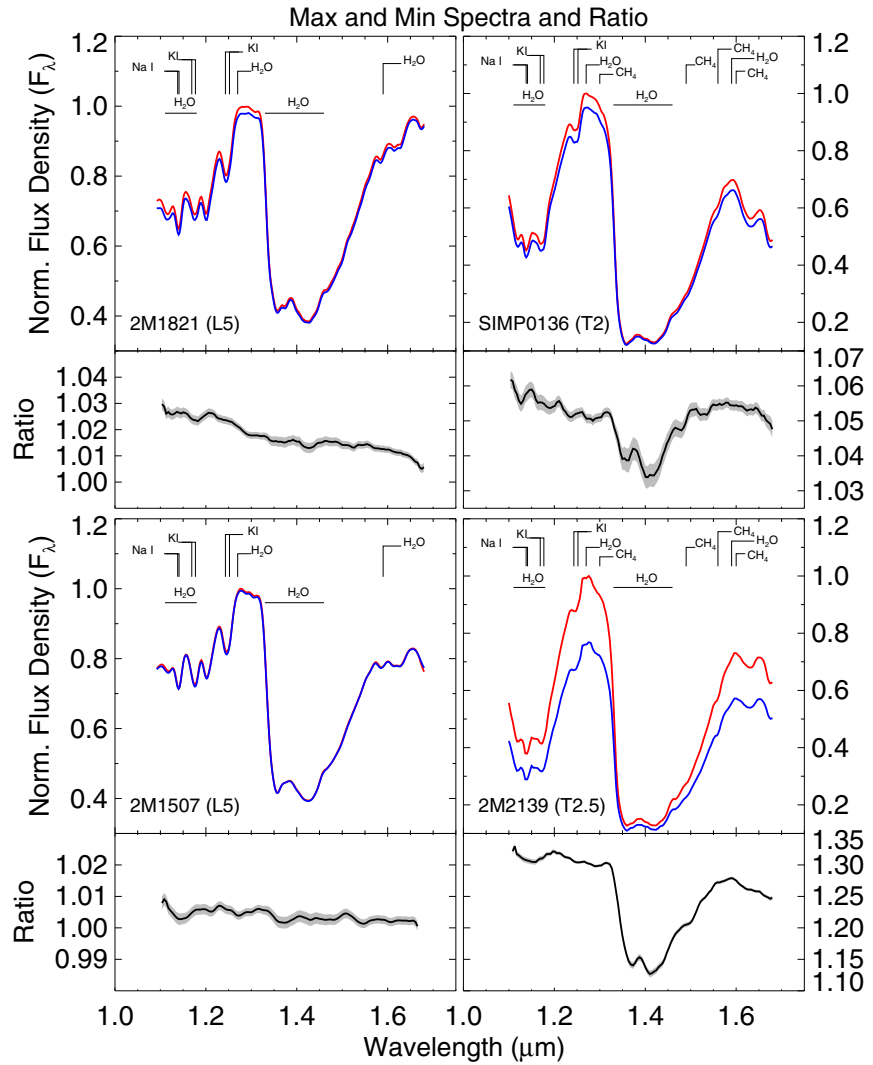

Figure 2. Brightest (red) and faintest (blue) spectra and their ratio (minor panels) during one $H S T$ visit for 2M1821, 2M1507, SIMP0136, and 2M2139, respectively. To increase the signal-to-noise, the brightest/faintest spectrum is from median combining the six brightest/faintest spectra from the same orbit. The gray band marks the uncertainty level in the ratio of the brightest and faintest spectra.

spectra shows generally weak wavelength dependence between 1.1 and $1.7 \mu \mathrm{m}$, and the water band around $1.4 \mu \mathrm{m}$ varies at similar amplitudes as the adjacent continuum.

To further illustrate the different behavior in the variability in and out of the $1.4 \mu \mathrm{m}$ water band, we perform synthetic photometry to measure the average flux density changes between the brightest and faintest spectra in several WFC3 medium bandpasses. We use the F139M filter to capture the average flux density in the water-absorption band and calculate the relative flux density change between the brightest and the faintest spectra, $(\Delta F / F)_{\text {In }}$. Similarly, we measure the flux density averaged over the F127M and F153M filters and calculate the relative variation in the average flux density out of the water-absorption band between the brightest and faintest spectra, $(\Delta F / F)_{\text {Out }}$. Then we take the ratio between the relative flux density changes in and out of the water band, $(\Delta F / F)_{\text {Out }} /(\Delta F / F)_{\text {In }}$. During the $H S T$ Visit 1 of $2 \mathrm{M} 1821$, e.g., the relative change in average flux density is $1.77 \% \pm 0.11 \%$ out of the water band and $1.54 \% \pm$ $0.21 \%$ in the water band, and the ratio of the two is $1.15 \pm 0.17$. As shown in Figure 3, the ratio $(\Delta F / F)_{\text {Out }} /(\Delta F / F)_{\text {In }}$ displays an increasing trend from the L5 dwarfs to the early T dwarfs. The L5 dwarfs show similar relative flux variation in and out of the water band, while the L/T dwarfs have greater relative flux change out of the water band. Such a trend with spectral types remains in observations of different epochs, even though the relative amplitudes of flux variation are different from visit to visit. The time between the two HST visits for targets in the Extrasolar Storms program is between one to three weeks, and for 
Relative Flux Density Change In and Out of Water Band

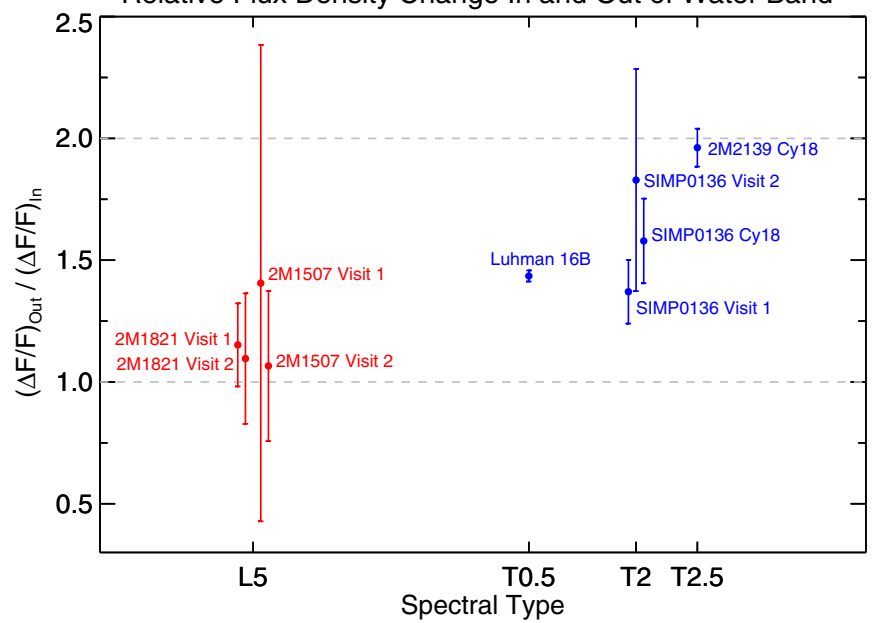

Figure 3. Ratio of relative flux density changes in and out of the water band between the brightest and faintest spectra for the two L5 dwarfs (red) and three $\mathrm{L} / \mathrm{T}$ transition dwarfs (blue). The relative flux density change in the water band, $(\Delta F / F)_{\text {In }}$, is the ratio of averaged flux densities over the WFC3 F139M bandpass, and the relative flux density change out of the water band, $(\Delta F / F)_{\text {Out }}$, is the ratio of averaged flux densities over both the WFC3 F127M and F153M bandpasses. The gray dashed lines mark where $(\Delta F / F)$ Out is the same as $(\Delta F / F)_{\text {In }}$ and twice the value of $(\Delta F / F)_{\text {In }}$, respectively. The values for Luhman 16B are calculated from data published in Buenzli et al. (2015). The relative flux variations out of the water band with respect to that in the water band shows an increasing trend from L5 dwarfs toward early T dwarfs.

SIMP0136, the observations from two HST cycles are separated by two years.

\section{DISCUSSION}

We investigate the near-IR spectral variability of the L5 dwarfs 2M1821 and 2M1507, and we find that the variations of the water-band absorption at $1.4 \mu \mathrm{m}$ exhibits pronounced differences between the two L5 dwarfs and two L/T transition dwarfs. We propose that such different behaviors could be due to the difference in the height of the dust particles in the atmospheres.

We propose a toy model that can quantitatively explain the observed behavior of the L5 and the L/T dwarfs. We assume that the intensity modulations, $\Delta I_{\text {int }}$, are introduced at an altitude, $z$, and that the dust layer causing the modulations is not emitting. The optical depth at $z$ measured from the top of the atmosphere is greater in the water band than in the adjacent continuum $\left(\tau_{\text {water }}>\tau_{\text {cont }}\right)$, but both are of the order of one. At a specific wavelength, $\lambda$, the modulations seen by the observer follow the Beer-Lambert law: $\Delta I_{\text {obs }}=\Delta I_{\text {int }} \cdot e^{-\tau_{\lambda}}$.

Then the relative variation in and out of the water band will be:

or $\epsilon=e^{-\left(\tau_{\text {water }}-\tau_{\text {cont }}\right)}$.

$$
\epsilon=\frac{\Delta I_{\mathrm{obs}, \text { water }}}{\Delta I_{\mathrm{obs}, \mathrm{cont}}}=\frac{\Delta I_{\mathrm{int}} \cdot e^{-\tau_{\mathrm{water}}}}{\Delta I_{\mathrm{int}} \cdot e^{-\tau_{\mathrm{cont}}}}
$$

In this scenario, if the modulations are introduced high in the atmosphere, the optical depth difference between in and out of water band will be negligible, leading to $\epsilon \sim 1$, as observed in the L5 dwarfs. If, however, the modulations are introduced deeper, the optical depth difference will be more significant, leading to reduced variability amplitude in the water band, as observed in L/T dwarfs. This simplistic model provides a correct relative variability amplitude difference between the continuum and water bands for both the L5 and the L/T cases.

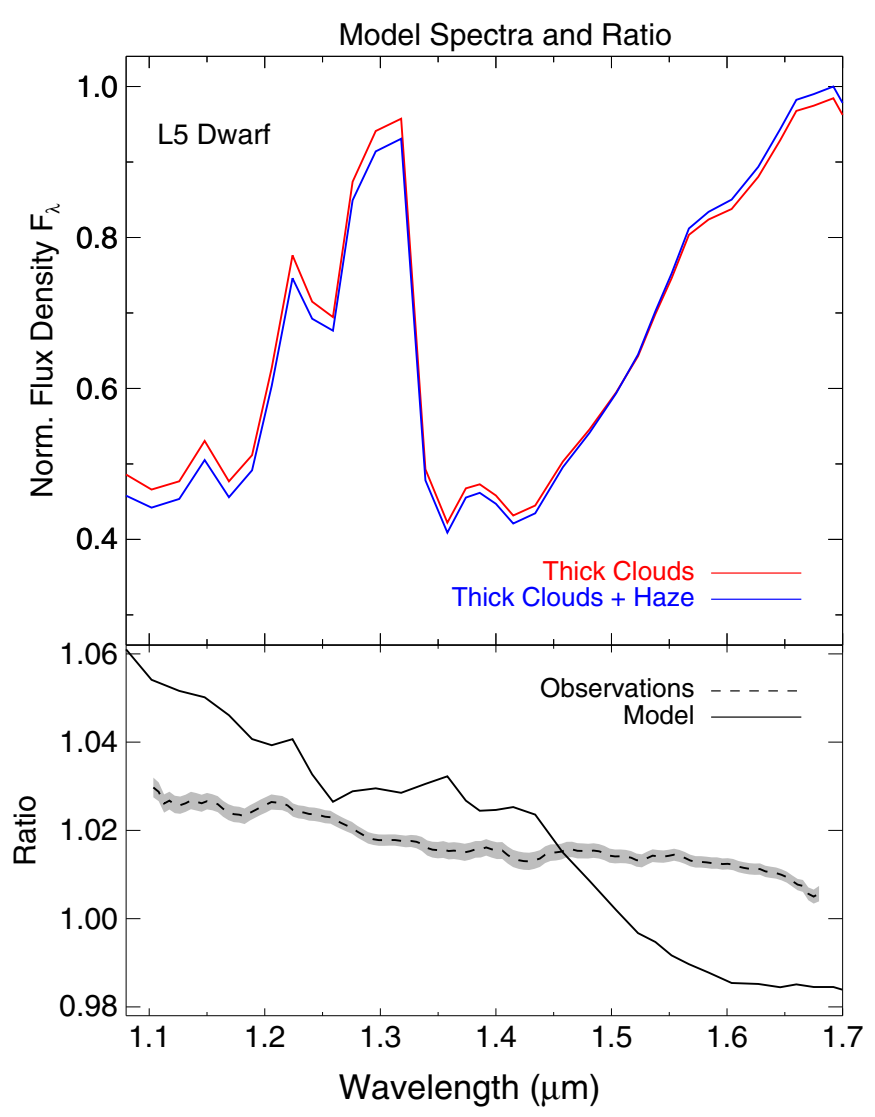

Figure 4. Model spectra and their ratios for an L5 dwarf based on the models of Saumon \& Marley (2008). The observed spectral ratio (dashed line) and associated uncertainties (gray band) of the L5 dwarf 2M1821 are also shown for comparison. With a haze layer high in the atmosphere, models of an L5 dwarf reproduce a qualitatively similar spectral flux ratio as observed with $2 \mathrm{M} 1821$ especially the similar variation amplitude in the $1.4 \mu \mathrm{m}$ water absorption band with the adjacent continuum.

When Looper et al. (2008) discovered 2M1821, they found several lines of evidence indicating an unusually dusty atmosphere, including unusually red slopes throughout the $Z, Y$, and $J$ band, red near-IR colors, weak $\mathrm{H}_{2} \mathrm{O}$ absorption, and silicate absorption at $9-11 \mu \mathrm{m}$. They considered either high clouds or high metallicity as the explanation for the high dust opacity. Looper et al. (2008) calculated a small tangential velocity for 2M1821, which suggests a young age. Therefore, 2M1821 could have low surface gravity (Gagné et al. 2014), which may explain the large condensate opacity high in the atmosphere (Marley et al. 2012). Our observations are consistent with such a scenario.

In an attempt to reproduce the spectral variability observed for 2M1821, we also explored a variety of model atmosphere cases (Saumon \& Marley 2008). By interpolating model spectra (Saumon \& Marley 2008) of different cloud thicknesses, Radigan et al. (2012) were able to predict the relative spectral variations of the early T dwarf 2M2139, including the low waterband amplitude. For 2M1821, we tried standard cloud models of Ackerman \& Marley (2001) with varying cloud thickness, but no combination/modification of existing cloud models could explain the wavelength dependence of the observed variability amplitude. To investigate if a spatially varying high-altitude haze might explain the observed variability, we compared two similar models. Both were for $T_{\text {eff }}=1800 \mathrm{~K}$ and $\log g=5$ with $f_{\text {sed }}=2$. We compared the emergent flux computed for a standard model with a model that additionally has a high, thin haze layer consisting of forsterite grains added to the top of 
the atmosphere. The haze particle size was set to have a single radius of $0.1 \mu \mathrm{m}$ and was confined to pressures less than 50 mbar. The column geometric optical thickness of this haze (the total $\tau$ of the haze were it composed of perfectly scattering particles of the same size) is 0.7. These haze particles are of smaller size than the dust particles in the condensate clouds and have much longer settling timescales. Figure 4 shows the ratio of the emergent flux of the two models in comparison with the observed spectral ratio, and we were able to produce the similar variability in and out of the water band with the haze model, further supporting the idea of dust particles residing high in the atmosphere of $2 \mathrm{M} 1821$.

While the shape of the spectral flux ratio is reproduced, we did not find a model flux ratio that is everywhere above unity as observed. The reason for this is that, when comparing two similar models, one with the high haze layer and one without, the model without the haze layer tends to always be cooler towards the top of the atmosphere. Thus the ratio of the two model spectra typically shows a flux excess (ratio $>1$ ) inside $J$ and $H$ bands, since the haze-free model allows more flux to escape within the window regions, which better sample the deeper and hotter parts of the atmosphere, but results in a flux deficit (ratio $<1$ ) inside of the water band as the less cloudy model is cooler. A more comprehensive model-fitting scheme will help better match the observed spectral ratio.

\section{CONCLUSION}

We have studied the near-IR spectral variability of the L5 dwarfs 2M1821 and 2M1507 for the first time and found that the variability of the $1.4 \mu \mathrm{m}$ water band can probe the height of the cloud covers in the atmospheres. The weak wavelength dependence of spectral variations observed on the L5 dwarfs indicates that the dust grains giving rise to the flux variability likely reside at high altitudes, and this also fits general model predictions. We have found that the relative amplitude of flux variability out of the $1.4 \mu \mathrm{m}$ water band compared with that in the water band displays an increasing trend from L5 dwarfs toward early T dwarfs. Additional observations of objects with a range of spectral types are required to further confirm this trend.

With a limited sample of a few objects, we have demonstrated that rotational spectral mapping can be used to probe different atmospheric depths with different spectral features. When more advanced observing facilities such as the James Webb Space Telescope become available, this technique can be applied to other atomic and molecular species and diagnose the atmospheric structures of brown dwarfs and directly imaged exoplanets in both horizontal and vertical directions. The high-altitude haze layers seen in brown dwarf atmospheres echo those found in exoplanetary atmospheres, further emphasizing the similarities in the properties of these ultracool atmospheres. Our observations open the possibilities of detailed comparative studies to understand the haze properties and behavior in both brown dwarf and exoplanetary atmospheres.

This work is part of the Spitzer Cycle-9 Exploration Program Extrasolar Storms. This work is based in part on observations made with the Spitzer Space Telescope, which is operated by the Jet Propulsion Laboratory, California Institute of Technology under a contract with NASA. Support for this work was provided by NASA through an award issued by JPL/Caltech. Support for HST GO programs 13176 and 13280.06-A was provided by NASA through a grant from the Space Telescope Science Institute, which is operated by the Association of Universities for Research in Astronomy, Inc., under NASA contract NAS526555. We acknowledge the outstanding help of Patricia Royle (STScI) and the Spitzer Science Center staff, especially Nancy Silbermann, for coordinating the HST and Spitzer observations. E.B. is supported by the Swiss National Science Foundation (SNSF).

\section{REFERENCES}

Ackerman, A. S., \& Marley, M. S. 2001, ApJ, 556, 872

Allard, F., Hauschildt, P. H., Alexander, D. R., Tamanai, A., \& Schweitzer, A. 2001, ApJ, 556, 357

Apai, D., Radigan, J., Buenzli, E., et al. 2013, ApJ, 768, 121

Artigau, É., Bouchard, S., Doyon, R., \& Lafrenière, D. 2009, ApJ, 701, 1534

Artigau, É., Doyon, R., Lafrenière, D., et al. 2006, ApJL, 651, L57

Biller, B. A., Crossfield, I. J. M., Mancini, L., et al. 2013, ApJL, 778, L10

Buenzli, E., Apai, D., Morley, C. V., et al. 2012, ApJL, 760, L31

Buenzli, E., Apai, D., Radigan, J., Reid, I. N., \& Flateau, D. 2014, ApJ, 782, 77

Buenzli, E., Saumon, D., Marley, M. S., et al. 2015, ApJ, in press (arXiv:1411.0003)

Burgasser, A. J., Gillon, M., Faherty, J. K., et al. 2014, ApJ, 785, 48

Burgasser, A. J., Marley, M. S., Ackerman, A. S., et al. 2002, ApJL, 571, L151

Burrows, A., Marley, M. S., \& Sharp, C. M. 2000, ApJ, 531, 438

Chabrier, G., Baraffe, I., Allard, F., \& Hauschildt, P. 2000, ApJ, 542, 464

Cohen, M., Wheaton, W. A., \& Megeath, S. T. 2003, AJ, 126, 1090

Crossfield, I. J. M., Biller, B., Schlieder, J. E., et al. 2014, Natur, 505, 654

Cushing, M. C., Roellig, T. L., Marley, M. S., et al. 2006, ApJ, 648, 614

Gagné, J., Lafrenière, D., Doyon, R., Malo, L., \& Artigau, É. 2014, ApJ, 783,121

Gillon, M., Triaud, A. H. M. J., Jehin, E., et al. 2013, A\&A, 555, L5

Heinze, A. N., Metchev, S., Apai, D., et al. 2013, ApJ, 767, 173

Helling, C., Woitke, P., \& Thi, W.-F. 2008, A\&A, 485, 547

Jones, H. R. A., \& Tsuji, T. 1997, ApJL, 480, L39

Knutson, H. A., Benneke, B., Deming, D., \& Homeier, D. 2014, Natur, 505, 66 Kostov, V., \& Apai, D. 2013, ApJ, 762, 47

Kreidberg, L., Bean, J. L., Désert, J.-M., et al. 2014, Natur, 505, 69

Lodders, K., \& Fegley, B., Jr. 2006, in Chemistry of Low Mass Substellar Objects ed. J. W. Mason (Chichester, UK: Praxis Publishing Ltd.), 1

Looper, D. L., Kirkpatrick, J. D., Cutri, R. M., et al. 2008, ApJ, 686, 528 Luhman, K. L. 2013, ApJL, 767, L1

Marley, M. S., Saumon, D., Cushing, M., et al. 2012, ApJ, 754, 135

Metchev, S., Heinze, A., Apai, D., et al. 2015, ApJ, in press (arXiv:1411.3051)

Morley, C. V., Fortney, J. J., Marley, M. S., et al. 2012, ApJ, 756, 172

Morley, C. V., Marley, M. S., Fortney, J. J., \& Lupu, R. 2014, ApJL, 789, L14

Radigan, J. 2015, ApJ, in press (arXiv:1408.5919)

Radigan, J., Jayawardhana, R., Lafrenière, D., et al. 2012, ApJ, 750, 105

Radigan, J., Lafrenière, D., Jayawardhana, R., \& Artigau, E. 2014, ApJ, 793, 75

Reid, I. N., Kirkpatrick, J. D., Gizis, J. E., et al. 2000, AJ, 119, 369

Robinson, T. D., \& Marley, M. S. 2014, ApJ, 785, 158

Saumon, D., \& Marley, M. S. 2008, ApJ, 689, 1327

Showman, A. P., \& Kaspi, Y. 2013, ApJ, 776, 85

Sing, D. K., Pont, F., Aigrain, S., et al. 2011, MNRAS, 416, 1443

Stephens, D. C., Leggett, S. K., Cushing, M. C., et al. 2009, ApJ, 702, 154

Tsuji, T. 2002, ApJ, 575, 264

Tsuji, T., Ohnaka, K., Aoki, W., \& Nakajima, T. 1996, A\&A, 308, L29

Wilson, P. A., Rajan, A., \& Patience, J. 2014, A\&A, 566, A111

Zhang, X., \& Showman, A. P. 2014, ApJL, 788, L6 